TRANSACTIONS OF THE

AMERICAN MATHEMATICAL SOCIETY

Volume 349, Number 12, December 1997, Pages 5031-5042

S 0002-9947(97)01885-0

\title{
COVERING SATO-LEVINE INVARIANTS
}

\author{
GUI-SONG LI
}

\begin{abstract}
Two covering versions of the Sato-Levine invariant are constructed which provide obstructions to certain two-component oriented links in the 3sphere being link concordant to boundary links. These covering invariants are rational functions one of which detects both nonamphicheirality and noninvertibility of oriented links.
\end{abstract}

\section{INTRODUCTION}

It has long been recognized that covering spaces of knot and link complements are of great importance in the study of classical knots and links. For over half a century, the various covering invariants have occupied a central place in the development of classical knot and link theory, especially in the study of difficult problems concerning amphicheirality, invertibility and concordance of knots and links.

In this paper we shall describe two new covering invariants for two-component links in the 3 -sphere. The first one, called the $\tau$-function, is a 2 -variable rational function over $\mathbf{Z}$ defined for links with vanishing Kojima $\eta$-function. The second one, called the $\delta$-function, is a 1-variable rational function over $\mathbf{Z}_{2}$ defined for links with vanishing $\tau$-function. Both rational functions are invariants of link concordance. Kojima's $\eta$-function [16] is a link concordance invariant for two-component links with vanishing linking number and may be viewed as a covering version of the classical linking number; our $\tau$-function and $\delta$-function may be viewed as covering versions of the Sato-Levine invariants [26] for three- and two-component links respectively and should best be called the covering Sato-Levine invariants of links.

The covering Sato-Levine invariants constructed in this paper provide obstructions to two-component links being concordant to boundary links, and both can be used to distinguish infinitely many different concordance classes of two-component links. However, the most striking property of the covering Sato-Levine invariants is that while the $\delta$-function is independent of one's choice of the orientation of a given link, the $\tau$-function is extremely sensitive to this and can be used to detect both nonamphicheirality and noninvertibility of two-component links even up to link concordance. This observation makes our $\tau$-function quite different from most of the commonly used link invariants, including the 2-variable Alexander polynomial [1], the Milnor invariants [23] and the various quantum group invariants constructed after Jones's discovery of his famous Jones polynomial [14]; all these invariants fail to distinguish two-component links from their inverses.

Received by the editors December 6, 1995 and, in revised form, June 13, 1996.

1991 Mathematics Subject Classification. Primary 57M25, 57N70, 57M10.

Key words and phrases. Links, link concordance, covering Sato-Levine invariants.

(C)1997 American Mathematical Society 
It should be noticed that if we are limited to two-component 1-trivial dichromatic links having vanishing linking numbers, or equivalently, to null-homotopic knots in the solid torus, then various covering invariants of this kind can easily be derived from known invariants for links in $S^{3}$. Among these covering invariants are those coming from Milnor's $\bar{\mu}$-invariants [23], which generalize both Kojima's $\eta$-function and our covering Sato-Levine invariants in this special case. These covering Milnor invariants are concordance invariants for null-homotopic knots in the solid torus, and detect both nonamphicheirality and noninvertibility of two-component links that are not detectable by Kojima's $\eta$-function nor by our $\tau$-function. The details will be given in another paper [20] of the author.

This paper is organized as follows. Section 1 contains basic definitions and conventions. In Section 2 a stable version of the Sato-Levine invariant is shown to be definable for semiboundary links in the universal abelian cover of any classical knot complement. This will be used in Section 3 to define the covering Sato-Levine invariants. We prove in Section 4 that these covering Sato-Levine invariants are link concordance invariants, and then construct in Section 5 examples, showing that the covering Sato-Levine invariants distinguish infinitely many different concordance classes of links and detect both nonamphicheirality and noninvertibility of links even up to link concordance.

\section{BASIC DEFINITIONS AND CONVENTIONS}

An $m$-component link is an ordered collection of $m$ disjoint oriented circles smoothly embedded in $S^{3}$. Two $m$-component links $L_{0}$ and $L_{1}$ are concordant if there exists an ordered collection $C$ of $m$ disjoint oriented annuli embedded smoothly and properly in $S^{3} \times I$ which restricts to $L_{0}$ and the inverse of $L_{1}$ over the boundaries. A Seifert surface for a knot or link is a compact oriented surface smoothly embedded in $S^{3}$ whose boundary is the given knot or link. A boundary link is one whose components bound disjoint Seifert surfaces. A semiboundary link is one each of whose components bounds a Seifert surface missing the other components.

For our purposes, we will also consider links in an arbitrary manifold, links with infinitely many components, and links whose components are disconnected. All of the above terminology makes sense for these generalized links. To avoid possible confusion, we use the convention that, whenever links are considered in a universal abelian cover, they are understood to be links in this broader context.

Throughout this paper all homology and cohomology groups are assumed to have untwisted integer coefficients.

\section{The Stable Sato-Levine invariant}

The Sato-Levine invariant is a concordance invariant for semiboundary links in euclidean space, which arises from the intersection of Seifert surfaces and provides an obstruction to a semiboundary link being concordant to a boundary link. This geometric invariant was discovered independently by Sato [26] and Levine (unpublished) and has since been studied much more extensively by Cochran [5, 6, 7], Orr [25] and Stein [27]. The main result of this section is an observation that a stable version of the Sato-Levine invariant can be defined for semiboundary links with possibly disconnected components in the universal abelian cover of the exterior of any classical knot. 
Suppose that a knot $K$ is given in $S^{3}$. We denote by $E=S^{3}-N(K)$ the exterior of $K$, where $N(K)$ is a small open tubular neighborhood of $K$ in $S^{3}$, and by $\widetilde{E}$ the universal abelian cover of $E$, which is oriented and framed by a pull-back of the standard orientation and framing of $S^{3}$.

Definition 2.1. Let $L=\left(K_{1}, K_{2}\right)$ be a two-component semiboundary link in $\widetilde{E}$ and let $\left(V_{1}, V_{2}\right)$ be a pair of transverse Seifert surfaces exhibiting $L$ as a semiboundary link. The stable Sato-Levine invariant $\tilde{\beta}(L)$ is the bordism class in $\pi_{1}^{S} \cong \mathbf{Z}_{2}$ represented by the intersection 1-manifold $V_{1} \cap V_{2}$ whose framing is induced by the framing of $\widetilde{E}$ and the normal vector fields of $V_{1}$ and $V_{2}$ in $\widetilde{E}$.

Definition 2.2. Let $L=\left(K_{1}, K_{2}, K_{3}\right)$ be a three-component semiboundary link in $\widetilde{E}$ and let $\left(V_{1}, V_{2}, V_{3}\right)$ be a triple of transverse Seifert surfaces exhibiting $L$ as a semiboundary link. Then each triple intersection point in $V_{1} \cap V_{2} \cap V_{3}$ inherits a sign from the ordering and orientations of the Seifert surfaces. The stable Sato-Levine invariant $\tilde{\beta}(L)$ is the algebraic sum of triple intersection points in $V_{1} \cap V_{2} \cap V_{3}$.

Theorem 2.3. The stable Sato-Levine invariant $\tilde{\beta}$ is well-defined for three- and two-component semiboundary links in $\widetilde{E}$.

Proof. By Milnor [24] the group $H_{2}(\widetilde{E})$ vanishes identically. It follows immediately that the stable Sato-Levine invariant for three-component links is independent of one's choice of transverse Seifert surfaces for links.

To show that the stable Sato-Levine invariant $\tilde{\beta}$ for two-component semiboundary links is well-defined, let $\left(V_{1}, V_{2}\right)$ and $\left(V_{1}^{\prime}, V_{2}^{\prime}\right)$ be two pairs of transverse Seifert surfaces for a given link $L=\left(K_{1}, K_{2}\right)$, both exhibiting $L$ as a semiboundary link. Then

$$
W_{i}=\left(V_{i} \times 0\right) \cup\left(K_{i} \times I\right) \cup\left(V_{i}^{\prime} \times 1\right), \quad i=1,2,
$$

are surfaces embedded smoothly in $\widetilde{E} \times I$ (after straightening the angles) whose normal bundles are trivial. We claim that both $W_{1}$ and $W_{2}$ bound compact oriented smooth 3 -submanifolds of $\widetilde{E} \times I$, denoted by $Z_{1}$ and $Z_{2}$ respectively, such that

$$
Z_{1} \cap\left(K_{2} \times I\right)=\left(K_{1} \times I\right) \cap Z_{2}=\emptyset
$$

and such that $Z_{1}$ meets $Z_{2}$ transversely. The result then follows since $Z_{1} \cap Z_{2}$ may be framed to establish a bordism between the stably framed 1-manifolds $V_{1} \cap V_{2}$ and $V_{1}^{\prime} \cap V_{2}^{\prime}$.

It remains to prove the claim. The proof will be modeled on the proof of a similar result of Sato [26] for semiboundary links in euclidean space, but the argument we use is along the same line as that used by Kirk and Koschorke [15], which is applicable for a much more general class of links called link maps. For our purpose we shall need the following result of Milnor [24], a generalization of which to noncompact homology circles could be found in Kojima and Yamasaki [16].

Lemma 2.4. Let $X$ be a compact smooth n-manifold which has the same homology type as a circle, and let $\tilde{X}$ be the universal abelian cover of $X$. Then the homology intersection pair $H_{k}(\widetilde{X}) \times H_{n-k}(\widetilde{X}, \partial \widetilde{X}) \rightarrow \mathbf{Z}$ vanishes identically for each $0<k<$ $n$.

To prove the claim, let us first notice that $W_{1}$ is null-homologous, since $H_{2}(\widetilde{E} \times I)$ vanishes identically. Now for each circle $l$ in $\widetilde{E} \times I$ disjoint from $W_{1}$, one may define the linking number between $l$ and $W_{1}$ to be the usual intersection number of $l$ 
with any $(n+1)$-chain bounded by $W_{1}$. It follows by Lemma 2.4 that this linking number is well-defined, hence induces a homomorphism from $H_{1}\left(\Sigma_{1}\right)$ to $\mathbf{Z}$, where $\Sigma_{1}=\widetilde{E} \times I-W_{1}$. This determines a cohomology class of $H^{1}\left(\Sigma_{1}\right)$, hence a map $f_{1}: \Sigma_{1} \rightarrow S^{1}$ via the Hopf-Whitney correspondence. Now pick a regular value $*$ of $f_{1}$. Then by transversality, the preimage $Z_{1}^{\prime}=f_{1}^{-1}(*)$ is a noncompact oriented smooth $(n+1)$-submanifold of $\Sigma_{1}$. Since each circle in $K_{2} \times I$ has a vanishing linking number with $W_{1}$, the regular value may be chosen so that $Z_{1}^{\prime}$ is disjoint from $K_{2} \times I$ by Sato [26]. Notice that $W_{1}$ has a trivial normal bundle. It follows by the proof of Proposition 2.2 in Kirk and Koschorke [15] that after a homotopy of $f_{1}$ within a small tubular neighborhood of $M$ in $\widetilde{E} \times I$, the boundary of the closure of $Z_{1}^{\prime}$ may be assumed to be $W_{1}$. Let $Z_{2}^{\prime}$ be defined similarly by replacing $W_{1}$ by $W_{2}$. Then the closures of $Z_{1}^{\prime}$ and $Z_{2}^{\prime}$ are the required 3-manifolds (Seifert manifolds) for $W_{1}$ and $W_{2}$ respectively.

This completes the proof of Theorem 2.3.

Remark 2.5. By Cochran [6] the Sato-Levine invariant for three-component links in $S^{3}$ is a link homotopy invariant and may be identified with the Milnor invariant $\bar{\mu}(123)$. A close examination of Cochran's proof of Lemma 5.4 in [6] convinces us that the stable Sato-Levine invariant for three-component links in $\widetilde{E}$ is again an invariant of link homotopy in which each (possibly disconnected) component of the link is allowed only to cross itself during the deformation. This observation may sometimes greatly simplify the computations.

\section{Covering Sato-Levine invariants}

Let $L=(M, K)$ be a two-component link in $S^{3}$ with vanishing linking number. We denote by $E$ the exterior of $K$ and by $\widetilde{E}$ the universal abelian cover of $E$ with $t$ the canonical generator of the group of infinite cyclic covering transformations determined by the given orientation of $K$. The action of $t$ on $\widetilde{E}$ induces a module structure of $H_{1}(\widetilde{E})$ over the Laurent polynomial ring $\Lambda=\mathbf{Z}[t, \bar{t}]$. It is a classical result in knot theory that $H_{1}(\widetilde{E})$ is an $\Lambda$-torsion; the Alexander polynomial of $K$ annihilates $H_{1}(\widetilde{E})$.

Since the linking number of $L$ is zero, the first component $M$ can be lifted to an oriented circle $u$ smoothly embedded in $\widetilde{E}$, and is covered by infinitely many embedded circles $t^{m} u$ where $m \in \mathbf{Z}$. The linking behaviour among these covering circles should definitely be rich sources of link invariants. However, the fundamental difficulty which has obstructed this approach is that the linking numbers between these circles are in general not even defined. This explains in part why most of the previous work on covering linking numbers dealt only with finite cyclic branched covers and the various metacyclic or metabelian branched covers (see $[4,12]$ and references therein). The solution is, however, fairly simple. Instead of $u$ one may consider a boundary cycle $\lambda(t) u$, where $\lambda(t)$ is any annihilator of $u$, and then try to get rid of $\lambda(t)$ in a suitable way to make the result well-defined. This idea has been applied by Kojima and Yamasaki [16] for the linking number in constructing the $\eta$-function, and our contribution is an observation that the same idea is equally applicable for the stable Sato-Levine invariant.

We start with the definition of Kojima's $\eta$-function and refer to [16] for more details. Let $L=(M, K)$ be a link in $S^{3}$ with vanishing linking number. We fix a lift

$u$ of $M$ to $\widetilde{E}$ and denote by $u_{0}$ the nearby lift of a parallel copy of $M$ in $S^{3}$ having a 
vanishing linking number. Suppose that $\lambda(t)$ is any nontrivial Laurent polynomial that annihilates $u$. Then Kojima's $\eta$-function of $L$ is a 1 -variable rational function over $\mathbf{Z}$ defined by

$$
\eta_{L}(x)=\frac{1}{\lambda(\bar{x})} \sum_{m}\left(u_{0} \cdot B_{m}\right) x^{m}
$$

where $B_{m}$ is any 2 -chain bounded by $t^{m} \lambda(t) u$ and "." is the intersection number.

Kojima's $\eta$-function is a well-defined link concordance invariant, which satisfies both $\eta_{L}(x)=\eta_{L}(\bar{x})$ and $\eta_{L}(1)=0$, and vanishes identically for boundary links by Kojima and Yamasaki [16]. This invariant is in fact completely determined by the 2-variable Alexander polynomial of $L$ together with the 1-variable Alexander polynomials of its two components by Jin [13], and is equivalent to Cochran's derived invariants defined in [6], hence vanishes identically if and only if all Milnor invariants of the form $\bar{\mu}(11 \ldots 1122)$ vanish by Stein [27]; see also Theorem 6.10 of Cochran [7]. In a recent paper [19] of the author, Kojima's $\eta$-function is generalized to be definable for certain admissible links in higher dimensions. It turns out that our generalized $\eta$-function often gives more information than Cochran's derived invariants for admissible links in higher dimensions.

Now replacing overlapped copies of $t^{m} u$ by nearby lifts of disjoint parallel copies of $M$ in $S^{3}$ having vanishing linking numbers if necessary, one may assume that $\left\{b_{m}\right\}$, where $b_{m}=t^{m} \lambda(t) u$ and $m \in \mathbf{Z}$, is a set of disjoint collections of disjoint circles embedded in $\widetilde{E}$, which we call a stable covering link for $L$. Suppose that the $\eta$-function of $L$ vanishes identically. Then each component $b_{m}$ of the stable covering link has a vanishing linking number with $t^{n} u$ for each $n \in \mathbf{Z}$, hence bounds an embedded surface missing the other components. In other words, the stable covering link $\left\{b_{m}\right\}$ is now a semiboundary link in the broader context; see Section 1. The construction in the previous section then applies.

Definition 3.1. Let $L=(M, K)$ be a two-component link in $S^{3}$ with vanishing $\eta$-function. The $\tau$-function of $L$ is a 2 -variable rational function over $\mathbf{Z}$ defined by

$$
\tau_{L}(x, y)=\frac{1}{\lambda(x y) \lambda(\bar{x}) \lambda(\bar{y})} \sum_{m, n} \tilde{\beta}\left(b_{0}, b_{m}, b_{n}\right) x^{m} y^{n},
$$

where $\lambda(t)$ is an annihilator of a lift $u$ of $M$ to $\widetilde{E}$ and $\left\{b_{m}\right\}$ is the corresponding stable covering link for $L$.

Definition 3.2. Let $L=(M, K)$ be a two-component link in $S^{3}$ with vanishing $\tau$-function. The $\delta$-function of $L$ is a 1 -variable rational function over $\mathbf{Z}_{2}$ defined by

$$
\delta_{L}(x)=\frac{1}{\lambda(x) \lambda(\bar{x})} \sum_{m} \tilde{\beta}\left(b_{0}, b_{m}\right) x^{m},
$$

where $\lambda(t)$ is an annihilator of a lift $u$ of $M$ to $\widetilde{E}$ and $\left\{b_{m}\right\}$ is the corresponding stable covering link for $L$.

We now come to the main result of this paper, whose proof will be given in the next section. It should be noticed that the above construction of the $\delta$-function makes sense for any two-component link with vanishing $\eta$-function. Nevertheless, we are only able to show that it is well-defined for two-component links with vanishing $\tau$-function.

Theorem 3.3. Both the $\tau$-function and the $\delta$-function are well-defined link concordance invariants which vanish for boundary links. 
That Kojima's $\eta$-function is additive under connected sum of two-component links was first noticed by Cochran [5], and our next result shows that the same property is shared by our covering Sato-Levine invariants. These results are of interest, since the connected sum operation is not even well-defined on the set of concordance classes of two-component links.

Definition 3.4. Let $L$ be a link in a 3-manifold $X$ and let $b=I \times I$ be an oriented band embedded in $X$ whose intersections with $L$ are along the $\operatorname{arcs}\{i\} \times I$ which lie in different components of $L$ and have opposite orientations to those inherited from that of $L$, where $i=0,1$. Removing the two $\operatorname{arcs}\{0,1\} \times I$ in $L$ and adding the two $\operatorname{arcs} I \times\{0,1\}$, one obtains a link with one less component, which is called an interior band summing of $L$. Now let $L_{0}=\left(M_{0}, K_{0}\right)$ and $L_{1}=\left(M_{1}, K_{1}\right)$ be two links in $S^{3}$ separated by the equator $S$, and let $b_{M}$ and $b_{K}$ be two disjoint oriented bands embedded in $S^{3}$, which connect $M_{i}$ 's and $K_{i}$ 's respectively, and each of which intersects $S$ in only one arc. Performing interior band summing via $b_{K}$ and $b_{M}$, one obtains a new two-component link $L_{0} \# L_{1}=\left(M_{0} \# M_{1}, K_{0} \# K_{1}\right)$, which is called a connected sum of $L_{0}$ and $L_{1}$. See Cochran [7].

Theorem 3.5. Both the $\tau$-function and the $\delta$-function (whenever definable) are additive under connected sum.

Proof. The theorem will be proved along the same line as that used in the proof of Theorem 4.1 of Cochran [5]. Let $W_{0}$ and $W_{1}$ be Seifert surfaces for $K_{0}$ and $K_{1}$ lying in $S_{0}-M_{0}$ and $S_{1}-M_{1}$ respectively, where $S_{i}$ is the hemisphere in which $K_{i}$ lies. Then $W_{0}$ and $W_{1}$ may be chosen to avoid the interiors of both $b_{M}$ and $b_{K}$. Indeed, for each intersection arc of $W_{i}$ with a band, one may remove an open disk in $W_{i}$ which contains that arc, and then add a tube along the band to connect-up to a small torus about $M_{i}$ or $K_{i}$. These tubes and tori may be nested to be mutually disjoint, and all selfintersection circles of the resulting $W_{i}$ may be eliminated by a sequence of "cut-and-paste" operations (see Theorem 4.1 in [5]). Now $W_{0} \cup b_{K} \cup W_{1}$ is seen to be a Seifert surface for $K_{0} \# K_{1}$ disjoint from $M_{0}, M_{1}$ and $b_{M}$. Construct the universal abelian cover $\widetilde{X}$ of the exterior of $K_{0} \# K_{1}$ using this Seifert surface, and let $u_{0}$ and $u_{1}$ be nearby lifts of $M_{0}$ and $M_{1}$ respectively. Then the interior band summing of $u_{0}$ and $u_{1}$ via a nearby lift of $b_{M}$ is a lift of $M_{0} \# M_{1}$ to $\widetilde{X}$. Let us pick now a nontrivial Laurent polynomial $\lambda(t)$ which annihilates both $u_{0}$ and $u_{1}$. Then the stable covering link for $L_{0} \# L_{1}$ is the interior band summing of stable covering links $\left\{b_{m}^{0}\right\}$ and $\left\{b_{m}^{1}\right\}$ for $L_{0}$ and $L_{1}$, where the bands are nearby lifts of suitably chosen parallel copies of $b_{M}$ in $S^{3}$. One may choose a collection of transverse Seifert surfaces exhibiting $\left\{b_{m}^{i}\right\}$ as a semiboundary link within the restriction of $\widetilde{X}$ over the interior of $S_{i}$ for $i=0,1$. These Seifert surfaces may again be chosen to avoid the bands. Since the unions of the resulting Seifert surfaces and the lifting bands are now Seifert surfaces for the stable covering link for $L_{0} \# L_{1}$, it follows readily that both the $\tau$-function and the $\delta$-function of $L_{0} \# L_{1}$ are the sums of that of $L_{0}$ and $L_{1}$, completing the proof of the theorem.

It should be noticed that Kojima's $\eta$-function is conjugate symmetric [16], hence can only be used to detect nonamphicheirality of two-component links up to link concordance. In Section 5 we will show by explicit examples that this is not always the case for the $\tau$-function. This observation often allows us to distinguish twocomponent links from their mirror images and inverses, a property that is not possessed by most of the known invariants of links. 
To see this, recall that an amphicheiral link is one which is equivalent to its mirror image obtained by composing with a reflection of $S^{3}$, and that an invertible link is one which is equivalent to its inverse obtained by reversing orientations of all its components. The existence of nonamphicheiral knots was first established by Dehn [9] as early as 1913, but it takes half a century before Trotter [29] proved the existence of noninvertible knots. Noninvertible knots up to concordance have been studied for example by Livingston [22] using the Casson-Gordon invariant. Although the existence of noninvertible knots guarantees the existence of noninvertible links, it is still of interest to have examples of noninvertible links with invertible proper sublinks; see Whitten [30], for example, and refer to Burde and Zieschang [4] for more references on this subject. The importance of studying this kind of problem may also be seen from the fact that, while the various quantum group invariants detect nonamphicheirality of a wide range of knots and links, they fail to distinguish a single knot or link from its inverse; see for example Birman [3]. Milnor's $\bar{\mu}$-invariants of weight $n$ can detect noninvertibility of links if $n$ is odd; however, they fail to detect noninvertibility of two-component links by Corollary (6.6) of Traldi [28].

Our $\tau$-function, on the other hand, is powerful in detecting nonamphicheirality and noninvertibility of two-component links. Indeed, suppose we are given a link $L=(M, K)$ in $S^{3}$ with vanishing linking number. Then it is readily seen that changing the orientation of $K$ corresponds to replacing the generator $t$ by $\bar{t}$, while changing the orientation of $M$ corresponds to changing the orientations of all lifting circles of $M$ in $\widetilde{E}$, and that changing the orientation of $S^{3}$ corresponds to replacing $t$ by $\bar{t}$ and changing the orientation of $\widetilde{E}$ simultaneously. Let the rational functions $\tau_{ \pm}(x, y)$ be defined by

$$
\tau_{ \pm}(x, y)=\tau(x, y) \pm \tau(\bar{x}, \bar{y}) .
$$

Then we immediately have the following result.

Theorem 3.6. Let $L=(M, K)$ be a two-component link in $S^{3}$ with vanishing $\eta$ function. If $L$ is amphicheiral (respectively invertible) up to link concordance, then the invariant $\tau_{+}(x, y)$ (respectively $\left.\tau_{-}(x, y)\right)$ vanishes identically.

It follows that $\tau_{ \pm}(x, y)$ in fact provide obstructions to two-component links being amphicheiral and invertible up to link concordance. In Section 5 we will see that the $\tau$-function is in general not conjugate symmetric. As a consequence, the invariants $\tau_{ \pm}(x, y)$ are in general not trivial, hence can really be used to distinguish twocomponent links from their mirror images and inverses.

\section{Proof of Theorem 3.3}

We prove in this section that both the $\tau$-function and $\delta$-function are well-defined link concordance invariants which vanish for boundary links. We shall use the same notations as in the above section. To begin with, let us first notice that the covering Sato-Levine invariants are independent of the choice of the lift $u$ of $M$ to $\widetilde{E}$ and vanish identically for boundary links. The independence of the choice of parallel copies of $M$ in $S^{3}$ with vanishing linking numbers follows since the stable Sato-Levine invariants are independent of the choice of Seifert surfaces.

To prove that the $\tau$-function is independent of one's choice of the annihilator $\lambda(t)$, notice that in the definition of the stable Sato-Levine invariant for threecomponent links, the Seifert surfaces involved may be relaxed to be only immersed 
and possibly disconnected. It follows that the Sato-Levine invariant is additive on the first component, that is,

$$
\tilde{\beta}\left(b_{i}+b_{j}, b_{m}, b_{n}\right)=\tilde{\beta}\left(b_{i}, b_{m}, b_{n}\right)+\tilde{\beta}\left(b_{j}, b_{m}, b_{n}\right)
$$

for all $i, j, m, n \in \mathbf{Z}$, and similarly on the second and third components. Now a simple argument shows that

$$
\sum_{m, n} \tilde{\beta}\left(b_{0}^{\prime}, b_{m}^{\prime}, b_{n}^{\prime}\right) x^{m} y^{n}=\mu(x y) \mu(\bar{x}) \mu(\bar{y}) \sum_{m, n} \tilde{\beta}\left(b_{0}, b_{m}, b_{n}\right) x^{m} y^{n}
$$

for each Laurent polynomial $\mu(t)$, where $b_{m}^{\prime}=\mu(t) b_{m}$. Hence the $\tau$-function is well-defined; see also the proof of Proposition 1 in Kojima and Yamasaki [16].

To prove that the $\delta$-function is well-defined, it suffices to verify that

$$
\tilde{\beta}\left(b_{i}+b_{j}, b_{m}\right)=\tilde{\beta}\left(b_{i}, b_{m}\right)+\tilde{\beta}\left(b_{j}, b_{m}\right)
$$

for all $i, j, m \in \mathbf{Z}$. In order to do this, suppose we are given a triple $\left(B_{i}, B_{j}, B_{m}\right)$ of transverse Seifert surfaces exhibiting $\left(b_{i}, b_{j}, b_{m}\right)$ as a semiboundary link. One may assume without changing the values of $\tilde{\beta}\left(b_{i}, b_{m}\right)$ and $\tilde{\beta}\left(b_{j}, b_{m}\right)$ that the framed embedded circles $c_{i m}=B_{i} \cap B_{m}$ and $c_{j m}=B_{j} \cap B_{m}$ are both connected. Since the link has a vanishing $\tau$-function, the intersection number of $c_{i m}$ and $c_{j m}$ in $B_{m}$ is zero. Remove the intersection points in $c_{i m} \cap c_{j m}$ pairwise by adding 1-handles to either $B_{i}$ or $B_{j}$ (see Banchoff [2], for example) to obtain an immersed surface which intersects $B_{m}$ in a collection $\left\{l_{r}\right\}$ of framed embedded circles, and then remove the selfintersection circles of the resulting immersed surface by a sequence of "cut-andpaste" operations to obtain a Seifert surface for $b_{i}+b_{j}$. The additivity then follows since

$$
\tilde{\beta}\left(b_{i}, b_{m}\right)+\tilde{\beta}\left(b_{j}, b_{m}\right)=\left[c_{i m}\right]+\left[c_{j m}\right]=\sum\left[l_{r}\right]=\tilde{\beta}\left(b_{i}+b_{j}, b_{m}\right),
$$

where [ ] denotes the framed bordism class of stably framed 1-manifolds. This proves that the $\delta$-function is well-defined.

It remains to show that the covering Sato-Levine invariants are link concordance invariants. To this end, let $C=(P, Q)$ be a concordance between two links $L_{0}=$ $\left(M_{0}, K_{0}\right)$ and $L_{1}=\left(M_{1}, K_{1}\right)$ which both have vanishing $\eta$-functions. Denote by $X$ the exterior of $Q$ in $S^{3} \times I$ and by $\widetilde{X}$ the universal abelian cover of $X$ with $t$ the canonical generator of the group of infinite cyclic covering transformations. Then $X$ restricts to the exterior $E_{i}$ of $K_{i}$ over $S^{3} \times i, \widetilde{X}$ restricts to the universal abelian cover $\widetilde{E}_{i}$ of $E_{i}$, and $t$ restricts to the given generator of the group of infinite cyclic covering transformations of $\widetilde{E}_{i}$ for $i=0,1$.

Let us fix a lift $w$ of $P \subset X$ to $\widetilde{X}$. Then $\partial w=u_{0}-u_{1}$, where $u_{i}$ is a lift of $M_{i} \subset E_{i}$ to $\widetilde{E}_{i}$. Since the annulus $P$ has a trivial normal vector bundle in $S^{3} \times I$, one may push $P$ along a normal vector field of $P$ in $S^{3} \times I$ to obtain a parallel copy $P^{\prime}$ disjoint from $P$. It should be noticed that the normal vector field one uses can in fact be chosen so that $P^{\prime}$ restricts to a parallel copy of $M_{0}$ having a vanishing linking number over $S^{3} \times 0$, and that once this is done, the restriction of $P^{\prime}$ to $S^{3} \times 1$ is a parallel copy of $M_{1}$ having a vanishing linking number, since the link concordance preserves the linking number. Now suppose we are given a nontrivial Laurent polynomial $\lambda(t)$ that annihilates both $u_{0}$ and $u_{1}$. Replacing overlapped copies of each $t^{m} w$ by nearby lifts of disjoint parallel copies of $P$ constructed as above, one may assume that $\left\{t^{m} \lambda(t) w\right\}$ is a set of disjoint collections of disjoint annuli smoothly embedded in $\widetilde{X}$ which restricts to the stable covering links $\left\{b_{m}^{0}\right\}$ and $\left\{b_{m}^{1}\right\}$ for $L_{0}$ and $L_{1}$ respectively over the boundaries. 
Now let $\left\{B_{m}^{i}\right\}$ be collections of transverse Seifert surfaces in $\widetilde{E}_{i}$ exhibiting $\left\{b_{m}^{i}\right\}$ as semiboundary links where $i=0,1$. Then one may assume that the embedded surface $W_{m}=B_{m}^{0}-t^{m} \lambda(t) w-B_{m}^{1}$ is null-homologous in $\widetilde{X}$ for each $m \in \mathbf{Z}$. To see this, notice that $X$ has the same homology type as a circle by Alexander duality. It follows by Levine [17] that $H_{2}(\widetilde{X})$ is a $\Lambda$-torsion and there indeed exists a nontrivial Laurent polynomial $\mu(t)$ which annihilates $W_{m}$ for each $m \in \mathbf{Z}$. Let $\lambda(t)$ be replaced by $\mu(t) \lambda(t)$ in the above. Then the resulting $W_{m}$ 's are all nullhomologous as desired, since both $H_{2}\left(\widetilde{E}_{0}\right)$ and $H_{2}\left(\widetilde{E}_{1}\right)$ vanish identically by Milnor [24].

We can now apply the same argument as in the proof of Theorem 2.3 to obtain a map $f_{m}: \widetilde{X}-W_{m} \rightarrow S^{1}$ for each $m \in \mathbf{Z}$ so that $Z_{m}=f_{m}^{-1}(*)$ is disjoint from $t^{n} \lambda(t) w$ whenever $m \neq n$ and the boundary of the closure of $Z_{m}$ is $W_{m}$ for each $m$, where $*$ is a suitably chosen regular value of $f_{m}$. It follows readily that the closure of $Z_{0} \cap Z_{m}$ may be naturally framed to establish a bordism between $B_{0}^{0} \cap B_{m}^{0}$ and $B_{0}^{1} \cap B_{m}^{1}$, and that the closure of $Z_{0} \cap Z_{m} \cap Z_{n}$ may be framed to establish a bordism between $B_{0}^{0} \cap B_{m}^{0} \cap B_{n}^{0}$ and $B_{0}^{1} \cap B_{m}^{1} \cap B_{n}^{1}$. This shows that the two covering SatoLevine invariants of $L_{0}$ and $L_{1}$ are both the same, completing the proof of Theorem 3.3 .

Remark 4.1. Notice that both the Sato-Levine invariant and Kojima's $\eta$-function are invariant under an even weaker equivalence relation called $I$-equivalence (see $[5,16])$ in which the concordances are only assumed to be topologically embedded. By the existence of normal vector bundles of locally flat topological submanifolds of 4-manifolds proved by Freedman and Quinn [10], the proof given above in fact shows that the covering Sato-Levine invariants are invariant under locally flat $I$ equivalence. But we do not know whether the local flatness could be dropped so that the covering Sato-Levine invarinats are invariant even under $I$-equivalence.

\section{EXAMPLES AND FURTHER REMARKS}

The computation of the covering Sato-Levine invariants is perhaps easiest when the component $K$ is unknotted; for in that case, $\widetilde{E}$ is homeomorphic to $\mathbf{R} \times D^{2} \subset \mathbf{R}^{3}$ and one can easily draw the liftings. Also, the annihilator $\lambda(t)$ may be taken to be 1 . Hence the $\tau$-function and the $\delta$-function are both finite Laurent polynomials. Moreover, the stable Sato-Levine invariant $\tilde{\beta}$ for covering links is now the standard (or modulo two) Sato-Levine invariant $\beta$ for links in $S^{3}$, which has been related to coefficients of the Conway polynomial for links by Cochran [6], and hence can be calculated even via Conway's recursive formula, at least up to sign.

We now construct examples showing that both the $\tau$-function and the $\delta$-function can be used to distinguish infinitely many different concordance classes of twocomponent links, and that the $\tau$-function can be used to detect both nonamphicheirality and noninvertibility of two-component links in $S^{3}$.

Example 5.1. Let $L(m, n)=(M, K)$ be a two-component link in $S^{3}$, where $m$ and $n$ are integers, $K$ is an unknotted circle in a 3-ball disjoint from the Borromean $\operatorname{ring} B$, and $M$ is obtained by adding two unknotted bands with $b_{x}$ and $b_{y}$ their oriented core arcs which connect two components of $B$ and link $K$ algebraically $m$ and $n$ times respectively; see Figure 1 . Notice that we have depicted the bands using their oriented core arcs and suppressed the data associated to the twistings in 


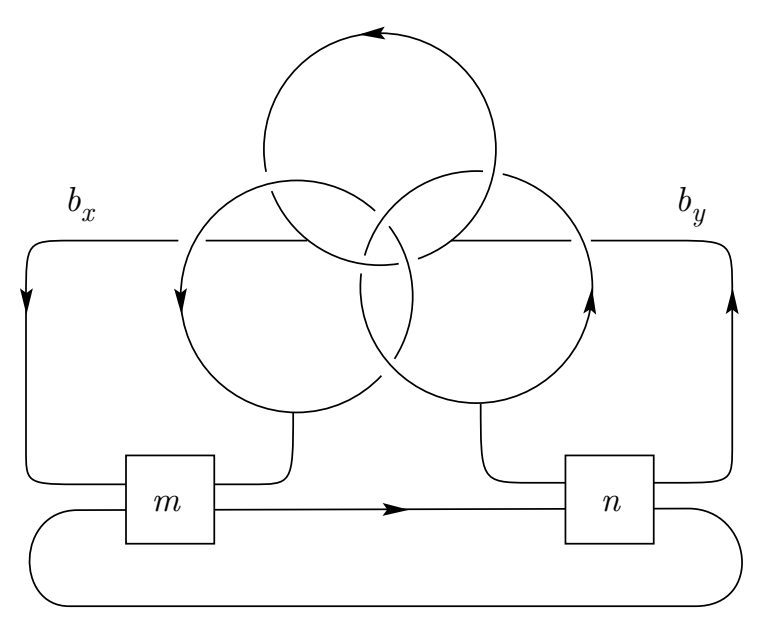

FiguRE 1

the bands which are irrelevant to the values of the covering Sato-Levine invariants. It is easily seen that the link $L(m, n)$ has a vanishing $\eta$-function.

Now one may check that if $m$ and $n$ are nonzero integers so that $m+n \neq 0$, then the $\tau$-function $\tau_{L}(x, y)$ of $L=L(m, n)$ is given by

$$
x^{m} \bar{y}^{n}-\bar{x}^{n} y^{m}+\bar{x}^{m+n} \bar{y}^{m}-\bar{x}^{m} \bar{y}^{m+n}+x^{n} y^{m+n}-x^{m+n} y^{n}
$$

since the Sato-Levine invariant of the Borromean ring $B$ is equal to 1 . Suppose that $m$ and $n$ are nonzero integers so that $m+n=0$, or one and only one of $m$ and $n$, say $m$, is nonzero. Then $L=L(m, n)$ has a vanishing $\tau$-function but a nonvanishing $\delta$-function, given by

$$
\delta_{L}(x) \equiv x^{m}+\bar{x}^{m} \bmod 2 .
$$

This follows since the Sato-Levine invariant of the Whitehead link is equal to 1 . Finally, if $m=n=0$, then both the $\tau$-function and the $\delta$-function of $L(m, n)$ vanish identically.

Notice that among the two-component links we have constructed, infinitely many are neither amphicheiral nor invertible, even up to link concordance. Indeed, if $m$ and $n$ are nonzero integers so that $m+n \neq 0$, then $L(m, n)$ has a nonvanishing $\tau_{-}$-function, hence is nonamphicheiral up to link concordance. On the other hand, if we assume further that both $m+2 n$ and $2 m+n$ are nonzero, then $L(m, n)$ has a nonvanishing $\tau_{+}$-function, hence is not concordant to its inverse. The details are omitted here.

Remark 5.2. By using the covering Milnor invariants defined in [20], further examples of nonamphicheiral and noninvertible two-component links in $S^{3}$ could be constructed which are not detectable by our $\tau$-function. The ideas can in fact be applied to construct for each $n>1$ infinitely many $n$-component links in $S^{3}$ which are neither amphicheiral nor invertible; among these examples infinitely many are even Brunnian links (see [21]).

Remark 5.3. By taking the connected sum, one may conclude by Example 5.1 that the covering Sato-Levine invariants may take as many finite Laurent polynomials as 
possible. However, the difficulties in calculating the stable Sato-Levine invariants in the covering spaces have prevented us from obtaining a complete realizability result for the covering Sato-Levine invariants. For the case of Kojima's $\eta$-function, a similar difficulty concerning the classical linking number has been overcome by Cochran [5], who showed that the $\eta$-function $\eta_{L}(x)$ may be expanded in positive powers of $y=(1-x)(1-\bar{x})$ and the coefficients of the resulting power series may be identified with his derived sequence of Sato-Levine invariants. In addition to its beauty and theoretical importance, such a reformulation is amenable to explicit calculations, inasmuch as it avoids the difficulties in constructing the infinite cyclic covers. It whould be interesting to know whether similar reformulations exist for the covering Sato-Levine invariants constructed in this paper.

Remark 5.4. It is an outstanding problem in link theory to determine which links are concordant to boundary links. The covering Sato-Levine invariants certainly provide obstructions to a link being concordant to a boundary link, and it would be interesting to know, as far as this problem is concerned, whether our covering SatoLevine invariants are ever stronger than Milnor's $\bar{\mu}$-invariants. Notice that the first examples of classical links which have vanishing $\bar{\mu}$-invariants but are not concordant to any boundary link were discovered by Cochran and Orr [8] using Blanchfield pairing on specific covering spaces. Different approaches using the Casson-Gordon invariant and the Atiyah-Patodi-Singer Index Theorem could be found in Gilmer and Livingston [11] and Levine [18] respectively. Although the $\tau$-function can be used to distinguish links having the same Milnor $\bar{\mu}$-invariants, it seems unlikely that the covering Sato-Levine invariants are well suited to distinguish links with vanishing $\bar{\mu}$-invariants from boundary links, as is seen by a computation of the $\tau$ functions for the examples given by the above authors; nevertheless, no proof for this has been found yet, and it remains still unknown whether the $\delta$-function can also be used to distinguish links having the same Milnor $\bar{\mu}$-invariants. It should be noticed that many of the results in this paper allow straightforward generalizations to define certain covering Sato-Levine invariants for two-component links in higher dimensions which again vanish for boundary links. Although no proof has been found yet, it seems unlikely that these covering Sato-Levine invariants could ever be nontrivial for links in higher dimensions, by the work of Orr [25].

\section{ACKNOWLEDGEMENTS}

I would like to express my gratitude to Hong Du for a useful discussion, and to Xiao-Song Lin for useful information concerning quantum group invariants and finite type invariants.

\section{REFERENCES}

1. J. Alexander, Topological invariants of knots and links, Trans. Amer. Math. Soc. 30 (1928), 275-306.

2. T. Banchoff, Triple points and surgery of immersed surfaces, Proc. Amer. Math. Soc. 46 (1974), 407-413. MR 51:14066

3. J. Birman, New points of view in knot theory, Bull. Amer. Math. Soc. 28 (1993), 253-287. MR 94b:57007

4. G. Burde and H. Zieschang, Knots, Walter de Gruyter, Berlin and New York, 1985. MR 87b:57004

5. T. Cochran, Geometric invariants of link cobordism, Comment. Math. Helv. 60 (1985), 291311. MR 87f:57021 
6. T. Cochran, Concordance invariance of coefficients of Conway's link polynomial, Invent. Math. 82 (1985), 527-541. MR 87c:57002

7. T. Cochran, Derivatives of links: Milnor's concordance invariants and Massey's products, Memoirs Amer. Math. Soc. 427 (1990). MR 91c:57005

8. T. Cochran and K. Orr, Not all links are concordant to boundary links, Bull. Amer. Math. Soc. 23 (1990), 99-106; Ann. of Math. 138 (1993), 519-554. MR 95c:57042

9. M. Dehn, Die beiden Kleeblattschlingen, Math. Ann. 75 (1914), 402-413.

10. M. Freedman and F. Quinn, Topology of 4-manifolds, Princeton University Press, Princeton, NJ, 1990. MR 94b:57021

11. P. Gilmer and C. Livingston, The Casson-Gordon invariant and link concordance, Topology 31 (1992), 475-492. MR 93h:57037

12. R. Hartley and K. Murasugi, Covering linkage invariants, Canadian J. Math. 29 (1977), 1312-1339. MR 56:16628

13. G. T. Jin, On Kojima's $\eta$-function of links, Lecture Notes in Mathematics, vol. 1350, SpringerVerlag, Berlin, Heidelberg and New York, 1988, pp. 14-30. MR 90c:57003

14. V. F. R. Jones, A polynomial invariant for knots via von Neumann algebra, Bull. Amer. Math. Soc. 12 (1985), 103-111. MR 86e:57006

15. P. Kirk and U. Koschorke, Generalized Seifert surfaces and linking numbers, Topology Appl. 42 (1991), 247-262. MR 92h:57038

16. S. Kojima and M. Yamasaki, Some new invariants of links, Invent. Math. 54 (1979), 213-228. MR 81b:57004

17. J. Levine, Knot modules I, Trans. Amer. Math. Soc. 229 (1977), 1-50. MR 57:1503

18. J. Levine, Link invariants via the eta invariants, Comment. Math. Helv. 60 (1994), 291-311. MR 95a:57009

19. G.-S. Li, Kojima's eta-function for manifold links in higher dimensions, Proc. Amer. Math. Soc. 125 (1997), 293-299. CMP 1997:2

20. G.-S. Li, Covering Milnor invariants for knots in the solid torus, preprint (1995).

21. G.-S. Li, Some noninvertible links, Proc. Amer. Math. Soc. (to appear).

22. C. Livingston, Knots which are not concordant to their inverses, Quart. J. Math. Oxford Ser. (2) 34 (1983), 323-328. MR 85d:57005

23. J. Milnor, Isotopy of links, Algebraic Geometry and Topology: A Symposium in Honor of S. Lefshetz, Princeton University Press, Princeton, NJ, 1957, pp. 280-306. MR 19:1070c

24. J. Milnor, Infinite cyclic coverings, Conference on the Topology of Manifolds, Prindle, Weber, and Schmit, Boston, MA, 1968, pp. 115-133. MR 39:3497

25. K. Orr, New link invariants and applications, Comment. Math. Helv. 62 (1987), 542-560. MR 89a: 57029

26. N. Sato, Cobordisms of semi-boundary links, Topology Appl. 18 (1984), 225-234. MR 86d: 57010

27. D. Stein, Massey products in the cohomology of groups with applications to link theory, Ph.D. Thesis, Brandeis University, 1985.

28. L. Traldi, Milnor's invariants and the completions of link modules, Trans. Amer. Math. Soc. 284 (1984), 401-424. MR 85j:57002

29. H. Trotter, Non-invertible knots exist, Topology 2 (1964), 275-280. MR 28:1618

30. W. Whitten, A pair of non-invertible links, Duke Math. J. 36 (1969), 695-698. MR 40:6532

Institute of Systems Science, Academia Sinica, Beijing 100080, China 